\title{
Stigmatisierungserfahrungen strafrechtlich verurteilter Männer im Reintegrationsprozess. Ergebnisse einer qualitativen Längsschnittstudie in der Schweiz
}

\author{
Franz Zahradnik
}

Eingegangen: 15. Juni 2021 / Angenommen: 7. Oktober 2021 / Online publiziert: 28. November 2021

(C) Der/die Autor(en) 2021

Zusammenfassung Strafrechtlich verurteilte Personen machen während einer Freiheitsstrafe sowie nach der Entlassung häufig Stigmatisierungserfahrungen, die den Reintegrationsprozess herausfordern und durchkreuzen können. Auf der Grundlage von Längsschnittinterviews mit strafrechtlich verurteilten Männern in der Schweiz werden deren subjektive Erfahrungen in Hinblick auf Stigmatisierung, die sie über mehrere Jahre hinweg in unterschiedlichen sozialen Kontexten gemacht haben, sowie ihre Verarbeitungsweisen derselben untersucht. In der kategorialen Auswertung des Gesamtsamples zeigt sich eine Korrespondenz von gehäuften Stigmatisierungserfahrungen und tendenziell sich stabilisierenden Reintegrationsverläufen. Es deutet sich an, dass gerade diejenigen, die eine ausgeprägte Reintegrationsorientierung zeigen, Situationen mit Stigmatisierungspotenzial etwa im Bereich der Arbeits- und Wohnungssuche nicht von vornherein auszuweichen versuchen, sondern diesen aktiv entgegentreten. Die dauerhafte Bewältigung solch herausfordernder und teils diskreditierender Situationen ist aber voraussetzungsvoll und gelingt nicht allen interviewten Männern gleichermaßen. Des Weiteren wird herausgearbeitet, welche Stigmatisierungserfahrungen die Interviewten in unterschiedlichen sozialen Kontexten und Phasen des Reintegrationsprozesses machen. Dabei zeigt sich, dass Stigmatisierungen nicht immer offen zutage treten, sondern von den Interviewten häufig antizipiert werden. Aktenkundige Stigmaeigenschaften hingegen werden dann offensichtlich, wenn ein Strafregisterauszug vorgelegt werden muss. Die damit verbundene Diskriminierung aber bleibt auf der Interaktionsebene zumeist verdeckt.

Schlüsselwörter Delinquenz $\cdot$ Stigmatisierung $\cdot$ Reintegration $\cdot$ Qualitative Längsschnittuntersuchung

Franz Zahradnik $(\bowtie)$

Institut für Erziehungswissenschaft, Lehrstuhl Ausserschulische Bildung und Erziehung, Universität Zürich, Freiestraße 36, 8032 Zürich, Schweiz

E-Mail: franz.zahradnik@ife.uzh.ch 


\title{
Stigmatization Experiences of Criminally Convicted Men in the Reintegration Process. Results of a Qualitative Longitudinal Study in Switzerland
}

\begin{abstract}
Criminally convicted people often experience stigmatization during a prison sentence as well as after release, which can challenge and thwart the reintegration process. Based on longitudinal interviews with criminally convicted men in Switzerland, this study examines their subjective experiences of stigmatization over several years in different social contexts, as well as their ways of dealing with them. The categorical evaluation of the entire sample shows a correspondence between frequent experiences of stigmatization and a tendency toward stabilizing reintegration processes. There are indications that those with a strong orientation toward reintegration do not try to avoid situations with the potential for stigmatization from the outset, for example in the area of job and housing search, but actively confront them. However, overcoming such challenging and sometimes discrediting situations in the long term is a prerequisite and not all of the men interviewed succeeded in the same way. Furthermore, it will be worked out which stigmatization experiences the interviewees make in different social contexts and phases of the reintegration process. It becomes apparent that stigmatization is not always overt, but is often anticipated by the interviewees. On the other hand, stigmatizing characteristics that are on record become obvious when an extract from the criminal record has to be presented. The associated discrimination, however, remains mostly concealed at the level of interaction.
\end{abstract}

Keywords Delinquency - Stigmatization · Reintegration - Qualitative longitudinal reasearch

\section{Einleitung}

Die soziale Integration strafrechtlich verurteilter Personen nach einer Inhaftierung bzw. Ersatzmaßnahme ist insgesamt ein komplexes und herausforderndes Unterfangen (Matt 2014). Die Entlassenen machen zumeist die schmerzhafte Erfahrung, dass sich das Kapitel der Strafe noch nicht gänzlich geschlossen hat, sondern die gesellschaftliche Erwartung einer Wiedereingliederung noch vor ihnen liegt. Diesen Weg müssen sie im Schatten des Stigmas einer viele Jahre aktenkundigen Zwangsunterbringung antreten. Erving Goffman hat die „Gefängnishaft“ unter den Stigmatypus „individuelle Charakterfehler“, die z.B. als „Willensschwäche“ und „Unehrenhaftigkeit“" wahrgenommen werden, eingeordnet (1975, S. 12f.). Stigmaträger:innen werden von den „Normalen“ (ebd. S. 13) in diskriminierender Weise besondert, indem diese eine ideologisch gefärbte „Stigma-Theorie“ (ebd. S. 14) konstruieren, die die „Gefährdung durch den Stigmatisierten nachweisen soll“ (ebd.). Je nach Verurteilungsgrund variiert die Einschätzung von „Gefährlichkeit“, die der ehemals inhaftierten Person zugeschrieben wird. Aber auch die Thematisierung von Kriminalität im öffentlichen und politischen Diskurs - zumeist am Beispiel spektakulärer Einzelfälle - konturiert das Bild der Entlassenen mit und kann Konjunkturen der 
Skandalisierung bedingen. In der Schweiz etwa bestimmt der Fall eines sogenannten ,jugendlichen Intensivtäters“ (Naplava 2008), der mittlerweile das Erwachsenenalter erreicht hat und sich in Sicherungshaft befindet, seit Jahren die Schlagzeilen, wodurch vor allem Kritik an sozialpädagogisch ausgerichteten Behandlungssettings befeuert wird und Rufe nach härteren Strafen immer wieder lauter werden (Schranz 2015). Zudem wurde in der Schweiz inzwischen der gesamte Vollzugsprozess inklusive der Aufsicht durch die Bewährungsdienste unter dem konzeptionellen Dach „Risikoorientierter Sanktionenvollzug (ROS)“ zusammengefasst. Als zentrale Ziele werden benannt, die „Rückfälligkeit [zu] reduzieren“ und die „Risikosensibilisierung aller Beteiligten“ zu fördern (www.rosnet.ch). Vor diesem Hintergrund ist festzustellen, dass der soziale Empfangsraum für ehemals Inhaftierte auch in der Schweiz durch eine grundlegende Skepsis sowie negative Einstellungen vorstrukturiert ist und von einer kompletten Beseitigung des „,Strafmakel[s]“ (Matt 2014, S. 45), also einer umfassenden Rehabilitation nach Verbüßung der Haftstrafe nicht auszugehen ist. Verschärft wird diese Situation zumeist durch multiple soziale Problemlagen, die in vielen Fällen schon vor der Inhaftierung bestanden und durch diese noch einmal zugespitzt wurden (ebd. S. 46 f.).

In diesem Beitrag werden Stigmatisierungserfahrungen von strafrechtlich verurteilten Männern in der Schweiz untersucht, die eine Haftstrafe oder eine ersatzweise angeordnete Maßnahme verbüßt haben. Grundlage der Untersuchung sind qualitative Längsschnittinterviews, die über vier Erhebungswellen ausgehend von der Endphase der Unterbringung im Abstand von eineinhalb Jahren erhoben wurden. Im Zentrum steht die Frage, wie die Interviewten negative Zuschreibungen und moralische Werturteile wahrnehmen und erleben. Zudem soll herausgearbeitet werden, welche Bewältigungsstrategien sie verfolgen, um einen Umgang mit der erlebten bzw. antizipierten Geringschätzung zu finden, und welche Bedeutung sie diesen Erfahrungen in Bezug auf den Reintegrationsprozess zurechnen. Zuerst werden theoretische Überlegungen hinsichtlich der Stigmatisierung von strafrechtlich verurteilten und inhaftierten Personen angestellt sowie Ergebnisse der bisherigen Forschung präsentiert (2). Im nächsten Schritt wird ein kurzer Überblick des Samples geboten (3) und es werden anhand der kategorisierenden Auswertung erste Spuren im Material zur Korrespondenz von Stigmatisierungserfahrungen und Reintegrationsverläufen aufgezeigt (4). In der weiteren Analyse des Interviewmaterials werden gegliedert nach unterschiedlichen sozialen Kontexten, die den Reintegrationsprozess mitstrukturieren, subjektive Deutungs- und Verarbeitungsweisen genauer untersucht (5). Der Beitrag schließt mit einem Fazit, in dem die Untersuchungsergebnisse eingeordnet und weiterführende Überlegungen angestellt werden (6).

\section{Theoretische Überlegungen und Forschungsstand}

Ein Stigma ist eine „zutiefst diskreditierend[e]“ (Goffman 1975, S. 11) Eigenschaft und eine Stigmatisierung die Zuschreibung derselben. Eine strafrechtliche Verurteilung ist das Ergebnis eines Aushandlungsprozesses im Rahmen eines Gerichtsverfahrens, die sich im weiteren Verlauf als hartnäckiges Stigma erweisen kann (Pager 2003; Petersilia 2003; Visher und Travis 2003; LeBel 2008; LeBel et al. 2008; Matt 
2014; Pescosolido und Martin 2015). Eine Stigmatisierung begründet ein besonderes Missverhältnis zwischen virtualer und aktualer sozialer Identität, also zwischen zugeschriebenen und selbst wahrgenommenen Eigenschaften (Goffman 1975). Eine solche Inkongruenz zwischen Selbst- und Fremdkategorisierung, die sich auch als umfassende „Fehlkategorisierung“ (Hirschauer 2017, S. 41) darstellen kann, hat vor allem dann schwerwiegende Konsequenzen für die Betroffenen, wenn dabei ihre Handlungsmacht im Extremfall gegen Null tendiert und ihre vielfältigen Zugehörigkeiten auf nur noch eine reduziert werden: „Sie wird zu ihrem ,Masterstatus'. Stigmatisierungen sind hochselektive und totalisierende Kategorisierungen, die alles andere irrelevant machen, im Sinne einer die Differenziertheit einer Person vernichtenden Wahrnehmung“ (ebd. S. 47). Auf strafrechtlich Verurteilte übertragen kann dies bedeuten, dass sie entweder schon zum Zeitpunkt der Verurteilung die Zuschreibung „kriminell“" als nicht passend für sich empfunden haben oder sich aber im weiteren Verlauf als „Abbrecher“ begreifen (Stelly und Thomas 2004, S. 211). In beiden Fällen kann eine Reaktualisierung der Kategorisierung als „kriminell““ Statusverluste bedingen und das Selbstverständnis herausfordern (Pescosolido und Martin 2015, S. 91 f.).

Solche Kategorisierungen sind immer verbunden mit allgemeinen und umfangreichen Annahmen über eine weitergefasste Gruppe von Personen. Einzelnen Personen werden Zugehörigkeiten zu solchen Gruppen sowie damit verbundene Eigenschaften aufgrund von bestimmten „Stigma-Theorie[n]“ (Goffman 1975, S. 14) zugeschrieben, mittels derer der Grad und die Qualität der Abweichung sowie das Gefährdungspotenzial gegenüber der Normalgesellschaft bestimmt wird. Im alltäglichen Sprachgebrauch stehen für die Kategorie „Kriminelle:r“ noch weitere „Stigmatermini“ (ebd.) wie etwa „Gauner:in“ oder „Verbrecher:in“" zur Verfügung, die zusätzliche Charakterisierungen mit sich tragen und damit über die ursprünglich unterstellte Unvollkommenheit hinausweisen. Im Falle von „Kriminalität“ spielt zudem meist der Tatvorwurf bzw. der Verurteilungsgrund eine entscheidende Rolle bei der moralischen Bewertung durch die Vertreter:innen der Normalgesellschaft bzw. diejenigen, die sich ihr zugehörig fühlen. Dabei ist eine gewisse moralische Hierarchisierung bezüglich der unterstellten Tatbestände anzunehmen, also Mörder:innen werden wohl anders eingeordnet als Dieb:innen. Es bestehen hier aber durch Rechtfertigungen und Plausibilisierungen in bestimmten Situationen und Kontexten Aushandlungsspielräume durch die je spezifische narrative Positionierung der Angeklagten im kulturellen Kriminalitätsdiskurs (Sykes und Matza 1957; Scott und Lyman 1968; Dollinger 2018).

Medien prägen und verändern das Bild von „Kriminellen“ in der Öffentlichkeit, z. B. im Rahmen fiktionaler Spielfilme oder aber aufgrund journalistischer Darstellungsformen. In der Schweiz hat es z. B. in den vergangenen Jahren der Fall eines jungen Mannes, der als Intensivtäter kategorisiert wurde, durch die mediale Berichterstattung zu einer traurigen Berühmtheit gebracht. Ausgehend von einem Artikel in einer Boulevardzeitung, in dem die zu hohen Kosten des Resozialisierungsprogramms mit Schlagworten wie „Sozial-Wahn“ und „Therapie-Industrie“ skandalisiert wurden, nahm das Thema breiten Raum in den Medien ein (Schranz 2015). An dem jungen Mann wurde in den darauffolgenden Jahren die Figur des untherapierbaren jugendlichen Gewaltstraftäters im öffentlichen Diskurs auch durch die 
zunehmende Politisierung des Falls zugespitzt (Fürst 2018). Es handelt sich dabei um einen besonderen Fall einer narrativ strukturierten „Problemgeschichte“ (Dollinger 2021), die nicht nur die gesellschaftliche Perspektive auf das Thema Jugendkriminalität und dessen Bearbeitung verändert, sondern auch einen deutlichen Einfluss auf die Justiz- und Politikpraxis entwickelt hat (Hinterleitner 2018). Insgesamt lässt sich feststellen:

Problemwahrnehmungen, Deutungsmuster und Strafbedürfnisse bilden zusammen einen kriminalitätsbezogenen Einstellungskomplex, der die staatliche Kriminalpolitik mit beeinflusst und generell eine wichtige Rolle dabei spielt, wie eine Gesellschaft mit Kriminalität und Straftätern umgeht (Oberwittler 2012, S. 807).

Bei einer strafrechtlichen Verurteilung handelt es sich um ein Stigma, das nicht sofort erkennbar ist. Das heißt, dass strafrechtlich Verurteilte im sozialen Verkehr zwar nicht schon von vornherein diskreditiert, aber potenziell diskreditierbar sind. Solange das Stigma noch unentdeckt ist, besteht also grundsätzlich die Möglichkeit, den Umgang mit dieser Information zu steuern (Goffman 1975, S. 56f.). Ist das Stigma aber freiwillig oder gegen den eigenen Wunsch aufgedeckt worden, tritt das Informationsmanagement in den Hintergrund und das Managen von situativen Spannungen wird wichtiger (ebd. S. 128f.). Die Strategien des Stigmamanagements lassen sich auf einem Kontinuum von reaktiv bis proaktiv verorten, wobei sich diese im Zeitverlauf verändern können. Reaktive Strategien zielen dann etwa auf Verheimlichung, Vermeidung, Rückzug oder selektive Offenlegung. Dazwischenliegende Strategien fokussieren eher auf eine graduelle Offenlegung, selektive Zugehörigkeitsbekundungen, Versuche der Diskreditierung der vermeintlichen Diskreditierer sowie die Zurückweisung moralischer Attribuierungen. Proaktive Strategien stützen sich dann schließlich auf eine umfassendere und vorbeugende Offenlegung sowie ein Engagement im Bereich öffentlicher Bildungsarbeit und sozialer Bewegungen (Siegel et al. 1998; LeBel 2008). Hierbei ist noch darauf hinzuweisen, dass es einen Unterschied macht, ob Stigmatisierungen auf konkreten Erfahrungen aufbauen, also eine Fremdkategorisierung beziehungsweise Diskriminierung erlebt wurde, oder ob das Stigma vielmehr soweit internalisiert wurde, dass bestimmte Ausweichhandlungen aufgrund der Antizipation einer drohenden Stigmatisierung vorweggenommen werden (Corrigan und Watson 2002, S. 36; Gurr und Jungbauer-Gans 2017, S. 32).

Schließlich ist noch auf die Kontextabhängigkeit von Stigmata hinzuweisen, denn nicht in allen Lebensbereichen haben sie dieselben Konsequenzen (Goffman 1975, S. 11). So kann es etwa sein, dass eine Eigenschaft im Kreise der Familie keine Rolle spielt, da sie hier gar nicht durch eine Zuschreibung aktiviert wird, während sie in bestimmten behördlichen Kontexten beständig reaktualisiert wird und deshalb diskriminierend wirkt. Ebenso ist es möglich, dass eben die zugeschriebene Eigenschaft unter anderen Träger:innen als positiver Ausweis dient und soziale Anerkennung sichert. Zudem sind Stigmata auch immer von sozialen Wandlungsprozessen und kulturell-geographischen Spezifika geprägt (Link und Phelan 2001).

Die sozialen und strukturellen Herausforderungen, mit denen sich ehemals Inhaftierte nach der Entlassung konfrontiert sehen, sind weithin bekannt. Mit meist nur geringen finanziellen Möglichkeiten ausgestattet gestaltet sich vor allem die Su- 
che nach einer bezahlbaren Wohnung als schwierige Aufgabe (LeBel et al. 2008), insbesondere wenn die Vermieter:innen neben Einkommensnachweisen auch einen aktuellen Strafregisterauszug verlangen (Matt 2014). Ebenso verhält es sich mit der Suche nach einer existenzsichernden Erwerbstätigkeit, wobei noch die durch die Inhaftierung unterbrochene Arbeitserfahrung als Einschränkung hinzukommen kann (Western et al. 2001; Pager 2003; Visher und Travis 2003). Zudem bestehen schon vor der Inhaftierung häufig weitere Belastungen wie langer und intensiver Drogenkonsum, fehlende Qualifikationen, Obdachlosigkeit, Sozialhilfebezug etc., die durch eine Inhaftierung zumeist noch verschärft werden. Auch sie sind Gegenstand von gesellschaftlichen Kategorisierungsprozessen und können so multiple Stigmata oder Stigmakomplexe begründen, die im weiteren Verlauf als besonders hinderlich und quasi als zweite, unsichtbare Bestrafung nach der Inhaftierung erfahren werden (Matt 2014, S. 45; LeBel 2012).

In einer aktuellen US-amerikanischen Studie wurden die Zusammenhänge zwischen Wohnen, Stigma und Inhaftierung in einem qualitativen Längsschnittdesign untersucht (Keene et al. 2018). Hier zeigt sich, dass die Suche nach einer Wohnung eine der entscheidenden Herausforderungen bezüglich der Reintegrationsbemühungen der Befragten darstellt, wobei sie versuchen, den Status von selbstverantwortlichen Bürger:innen zu erreichen und die mit Inhaftierung, Armut und Wohnungslosigkeit verbundenen sowie wohnortbezogenen Stigmata abzustreifen. Die Suche nach einer Wohnung wird als beschwerliches Unterfangen beschrieben, da die Betroffenen mit vielfältigen strukturellen und interpersonellen Formen des Inhaftierungsstigmas konfrontiert werden. Im Sinne einer sich selbstverstärkenden Rückkopplung wird das Inhaftierungsstigma gestärkt und bestehende Ungleichheitsverhältnisse werden verschärft und zugleich legitimiert.

Ein weiterer wichtiger Faktor für den Reintegrationsprozess wird in der Akzeptanz der Person und ihrer Vorhaben durch Familie und soziales Umfeld gesehen (Visher und Travis 2003). Hier kann es aber zu Problemen kommen, wenn etwa das Stigma durch die Nachbarschaft auch auf die Familie übertragen wird und diese sich distanziert, um sich der unfreiwilligen Mithaftung zu entziehen (Matt 2014). Auch beim Aufbau einer Partnerschaft sowie neuer Freundschaften kann das Stigma der strafrechtlichen Verurteilung ein bedeutsames Hindernis darstellen. Soziale Kontakte bleiben dann nicht selten auf solche Kreise beschränkt, in denen eine Stigmatisierung nicht befürchtet werden muss (ebd.).

Neben reaktiven Bewältigungsstrategien wie sozialem Rückzug wurden bei ehemals Inhaftierten auch proaktive Strategien beobachtet. So kann einer Kriminalisierung der eigenen Person auch entgegengetreten werden, indem auf entschuldigende und rechtfertigende Erklärungsmuster für das zugeschriebene Fehlverhalten rekurriert wird (Stenger 1985). Eine weitere Reaktionsweise besteht in der Betonung eines veränderten Selbst, das sich über soziales Engagement einbringen möchte. Im Sinne eines „making good“ (Maruna 2001) oder ,giving back“ (Visher und Travis 2003) kann etwa die Rolle eines Mentors für andere stigmatisierte Personen wie Inhaftierte oder Drogenkonsument:innen eingenommen werden, um die eigenen Erfahrungen als ,professional ex-“" weiterzugeben (LeBel 2008). 


\section{Methodische Anlage der qualitativen Längsschnittuntersuchung}

Die Daten dieser Untersuchung entstammen dem durch den Schweizerischen Nationalfonds (SNF) geförderten Forschungsprojekt „Wege aus der Straffälligkeit Reintegration verurteilter Straftäter“, das seit 2013 am Institut für Erziehungswissenschaft der Universität Zürich durchgeführt wird (Humm et al. 2022). Zielstellung der Studie ist es, eine breitere Wissensbasis hinsichtlich der Dynamiken von Reintegrationsprozessen über einen längeren Zeitraum zu gewinnen, weshalb ein qualitatives Längsschnittdesign gewählt wurde. Anfangs wurden problemzentrierte Interviews (Witzel 2000) mit 50 Männern erhoben, die sich möglichst in der Endphase einer strafrechtlich verfügten Zwangsunterbringung in einer Haftanstalt oder Ersatzmaßnahme befanden, um schon in dieser frühen Phase des Reintegrationsprozesses ihre Zukunftsentwürfe in den Blick zu bekommen. Sowohl hinsichtlich des Alters der Interviewpartner, das zwischen 17 und 61 Jahre lag, als auch der Verurteilungsgründe, die von Bagatell- bis hin zu Tötungsdelikten reichten, konnte eine heterogene Samplestruktur realisiert werden. Mit 30 der Männer konnten im Abstand von etwa eineinhalb Jahren insgesamt vier Interviews geführt werden, so dass nun jeweils ein Zeitraum von viereinhalb bis fünf Jahren in den Blick genommen werden kann. Diese insgesamt 120 Interviews bilden die Grundlage der nachfolgenden Analysen.

Die Auswertung des umfänglichen Datenmaterials wurde einerseits durch kategorisierende Verfahren in Anlehnung an die Grounded Theory Methodology (Strauss 2007) organisiert, andererseits wurden aber auch ausführliche Einzelfallanalysen erstellt. Die Entwicklung eines Kategoriensystems wurde von theoretischen Überlegungen und Erkenntnissen aus anderen Untersuchungen inspiriert, zum anderen wurden ausgehend von offenen Kodierschritten neue Konzepte erarbeitet oder bestehende ergänzt und verfeinert. Als zentrale Kategorien erwiesen sich u. a. die Bedeutung der eigenen Straffälligkeit, formelle und informelle Beziehungen, Erwerbsarbeit, Drogenkonsum, Schulden, Gesundheit und Stigmatisierungserfahrungen. Um die Relevanz der unterschiedlichen Kategorien im Längsschnitt einordnen zu können, wurden deren Ausprägungen hinsichtlich Kontinuitäten und Veränderungen im Zeitverlauf untersucht. Es ließen sich so drei verdichtete Längsschnitt-Kategorien in Bezug auf den Reintegrationsprozess bilden: (a) eine kontinuierlich stabilisierende Entwicklung, (b) eine begrenzt stabilisierende Entwicklung und (c) eine stagnierende Entwicklung (Rieker 2022). In ausführlichen Fallanalysen wurden schließlich spezifische Aspekte, die besonders aufschlussreich erschienen, mittels sequenz- und positionierungsanalytischer Auswertungsverfahren genauer untersucht (Soeffner 2004; Lucius-Hoene und Deppermann 2004).

Im Folgenden wird nun die gesamte Untersuchungsgruppe in Hinblick auf die Korrespondenzen von Stigmatisierungserfahrungen und Reintegrationsverläufen betrachtet. Anschließend werden einzelne Interviewpassagen genauer analysiert, um deren Bedeutungen für unterschiedliche Phasen und Kontexte des Reintegrationsprozesses herauszuarbeiten. 


\section{Betrachtung der gesamten Untersuchungsgruppe}

Im Zuge der Auswertung des Gesamtsamples wurden vorerst vier Kategorien in Hinblick auf Stigmatisierungserfahrungen gebildet, die auf Konzepten der Stigmatisierungsforschung aufbauen (vgl. Abschnitt 2). So wurde vor allem der Unterscheidung von erlebter und antizipierter Stigmatisierung Rechnung getragen, indem zuerst Textstellen kodiert wurden, in denen konkrete Situationen erlebter Abwertungen aufgrund der strafrechtlichen Verurteilung geschildert werden. Dabei wurde unterschieden zwischen ,Stigmatisierung im Kontext von Arbeit“, etwa durch Vorgesetzte, und „Stigmatisierung außerhalb von Arbeit“, z. B. durch Betreuungspersonen in Maßnahmen, durch Vermieter:innen bei der Wohnungssuche oder im privaten Umfeld. Als „Erwartete Stigmatisierung“ wurden solche Textstellen kodiert, die sich zwar ebenfalls auf die genannten Kontexte beziehen, in denen aber keine konkret erlebten Situationen erkennbar sind, sondern Stigmatisierungen von den Teilnehmern antizipiert wurden, also z.B. als Grund für die Ablehnung einer Bewerbung die frühere Verurteilung vermutet oder dies für bevorstehende Situationen als ausschlaggebender Faktor erwartet wurde. Schließlich gab es noch die Fälle, bei denen „keine Thematisierung“ von Stigmatisierungserfahrungen stattgefunden hat.

Aussagen zu Stigmatisierungserfahrungen wurden in den Interviews mit 26 der 30 Männer gefunden. Das heißt, dass vier der Männer in keinem der vier Interviews von solchen Erfahrungen berichtet haben, während dies bei den anderen mindestens einmal der Fall war. Zudem waren in den Fällen, bei denen Stigmatisierungserfahrungen erkennbar wurden, Mehrfachnennungen möglich. In der Übersicht lassen sich einige Tendenzen bezüglich der Verteilung von Stigmatisierungserfahrungen erkennen. Zuerst ist die über die Wellen hinweg relativ hohe Anzahl an NichtThematisierungen bemerkenswert. Hier sind keine Mehrfachnennungen möglich, es handelt sich also um Fälle und nicht um die Anzahl von Kodierungen. In der ersten Welle z. B. haben 18 Personen keinerlei Stigmatisierungserfahrungen berichtet, während sich die 16 Nennungen von Stigmatisierungserfahrungen auf zwölf Personen verteilen (Tab. 1).

Die wenigen arbeitsbezogenen Stigmatisierungserfahrungen in der ersten Welle lassen sich wohl darauf zurückführen, dass die meisten Teilnehmer zu diesem Zeitpunkt noch in Institutionen des Vollzugs oder der Nachbetreuung untergebracht waren und nur wenige schon außerhalb der Einrichtungen in Kontakt mit dem Arbeits- und Wohnungsmarkt gekommen waren. Der Anstieg in der zweiten Welle lässt sich als Folge der zuvor erfolgten Austritte aus den Einrichtungen und der intensivierten Arbeitssuche interpretieren. Dies wird durch die vergleichsweise niedrige

Tab. 1 Verteilung der Stigmatisierungserfahrungen

\begin{tabular}{llllll}
\hline & $\begin{array}{l}\text { Stigmatisierung im } \\
\text { Kontext von Arbeit }\end{array}$ & $\begin{array}{l}\text { Stigmatisierung au- } \\
\text { Berhalb von Arbeit }\end{array}$ & $\begin{array}{l}\text { Erwartete Stig- } \\
\text { matisierung }\end{array}$ & $\begin{array}{l}\text { Keine The- } \\
\text { matisierung }\end{array}$ & Summe \\
\hline W1 & 4 & 5 & 7 & 18 & 34 \\
W2 & 8 & 6 & 7 & 10 & 31 \\
W3 & 3 & 7 & 8 & 15 & 33 \\
W4 & 1 & 4 & 5 & 22 & 32 \\
Summe & 16 & 22 & 27 & 65 & $(130)$ \\
\hline
\end{tabular}


Tab. 2 Häufigkeit der Stigmatisierungserfahrungen und Reintegrationsverlauf

\begin{tabular}{lllll}
\hline & 3 oder 4 Wellen & 1 oder 2 Wellen & $\begin{array}{l}\text { Keine Thematisie- } \\
\text { rung }\end{array}$ & Summe \\
\hline Stabilisierung & 5 & 9 & 3 & 17 \\
Begrenzte Stabilisierung & 3 & 2 & 1 & 6 \\
Stagnation & 1 & 6 & - & 7 \\
Summe & 9 & 17 & 4 & $(30)$ \\
\hline
\end{tabular}

Anzahl an Nicht-Thematisierungen in der zweiten Welle gestützt. Ansonsten fallen die allgemein niedrigen Zahlen der Stigmatisierungserfahrungen in der vierten Welle auf, die auf einen moderaten Integrationseffekt im Sinne einer abnehmenden Bedeutung diskreditierbarer Eigenschaften hindeuten. Alternative Erklärungen könnten aber auch auf die Gewöhnung an abwertende Fremdzuschreibungen oder resignative Tendenzen bei den Betroffenen abstellen.

Nimmt man in den Blick, inwiefern Stigmatisierungserfahrungen mit den fallbezogenen Reintegrationsverläufen in Zusammenhang stehen, dann fällt auf, dass vor allem diejenigen, die über die Wellen hinweg tendenziell einen stabilisierenden Verlauf aufweisen, solche Erfahrungen berichten. Dabei zeigt sich, dass von den 14 Personen mit stabilisierendem Reintegrationsverlauf, die von Stigmatisierungserfahrungen berichten, bei fünf Personen solche Erfahrungen in mindestens drei Wellen festgestellt wurden. Dies könnte damit in Verbindung stehen, dass sie sich gegebenenfalls häufiger in Kontexten bewegen, in denen die strafrechtliche Verurteilung ein Thema werden kann (Tab. 2).

Auch bei denjenigen mit einem begrenzt stabilisierten Reintegrationsverlauf werden gehäufte Stigmatisierungserfahrungen im Reintegrationsprozess deutlich. Im Falle eines stagnierenden Reintegrationsverlaufs hingegen fällt auf, dass dort häufiger nur in ein oder zwei Wellen solche Erfahrungen ersichtlich werden. In diesen Fällen ist zumeist über längere Zeit eine zum Teil enge Anbindung an betreuende Einrichtungen gegeben, die eine Art Schutzraum vor Kontexten bieten können, in denen man potenziell häufiger mit abwertenden Zuschreibungen konfrontiert wird. Zudem kann Stigmatisierung gerade auch dann wirksam sein, wenn sie nicht direkt erfahren wird, sondern ihr durch Vermeidung und Rückzug auszuweichen versucht wird.

Insgesamt stellen sich Stigmatisierungserfahrungen schon vor dem Hintergrund der kategorisierenden Auswertung als ein vielschichtiges Phänomen dar. Im Weiteren gilt es weiter aufzuschlüsseln, welche feineren Ausprägungen sich hinter dem Unterschied von erwarteter und erlebter Stigmatisierung sowie der Korrespondenz von stabilisiertem Reintegrationsverlauf und mehrfacher Stigmatisierungserfahrungen verbergen.

\section{Stigmatisierungserfahrungen in unterschiedlichen Phasen und Kontexten des Reintegrationsprozesses}

Wenn man die Stigmatisierungserfahrungen im Gesamtsample in den Blick nimmt, dann zeigt sich eine sehr große Variationsbreite hinsichtlich ihrer Erscheinungsfor- 
men sowie Reaktionsweisen in unterschiedlichen Phasen und Kontexten des Reintegrationsprozesses. Die folgende Ergebnisdarstellung ist deshalb nicht mehr nur an den oben beschriebenen, die breitere Auswertung des Materials anleitenden vier Kategorien ausgerichtet. Vielmehr steht an erster Stelle eine zeitlich gegliederte Aufteilung, die jeweils in sich nach unterschiedlichen Kontexten organisiert ist. Zuerst werden die Erzählungen in Hinblick auf die Unterbringung und Behandlung in verschiedenen Vollzugskontexten untersucht (5.1). Daran anschließend wird es um Erzählungen gehen, die sich auf die Zeit nach der Entlassung beziehen (5.2), und zwar differenziert nach Stigmatisierungserfahrungen im Kontext der Betreuung durch die Bewährungsdienste (5.2.1) und der Integration in Arbeit (5.2.2) sowie in weiteren sozialen Kontexten wie Wohnungssuche, Behördenkontakte, soziale Kontakte und Beziehungen (5.2.3).

\subsection{Stigmatisierungserfahrungen im Vollzugskontext}

Die Interviewten berichten in Hinblick auf die Zeit der strafrechtlich angeordneten Unterbringung von Stigmatisierungserfahrungen in unterschiedlichen Ausprägungen. Während diejenigen, die eine Haftstrafe verbüßten, eher nicht von solchen Erfahrungen berichten, ist dies bei Maßnahmen zur Behandlung von psychischen Störungen sowie Suchtbehandlung selten, aber für Schutzmaßnahmen und Maßnahmen für junge Erwachsene häufiger der Fall. Dieses Ungleichgewicht könnte damit in Zusammenhang stehen, dass sich die jungen Männer ohnehin in einer konflikthaften und krisenanfälligen Lebensphase befinden und der Erziehungsauftrag in Maßnahmen für diese Altersgruppe in einem hochstrukturierten und rigiden pädagogischen sowie therapeutischen Rahmen verfolgt wird (Zahradnik und Humm 2016, 2019).

\subsubsection{Schutzmaßnahmen und Maßnahmen für junge Erwachsene}

In den Erzählungen der jungen Männer, die in Schutzmaßnahmen oder Maßnahmen für junge Erwachsene untergebracht sind, finden sich unterschiedliche Ausführungen zu Stigmatisierungserfahrungen. Meistens beziehen sich diese auf das Verhältnis zu den betreuenden Fachkräften, deren Handeln stellenweise als herabwürdigend erlebt wird, wenngleich in vielen Fällen auch positive Beziehungserfahrungen geschildert werden (Zahradnik et al. 2019; Zahradnik und Humm 2016, 2019).

$\operatorname{Marcel}^{1}(\mathrm{a})^{2}$ zum Beispiel schildert negative Erfahrungen im Therapiebereich der Einrichtung. Er habe sich nicht als ganze Person wahrgenommen gefühlt, sondern vor allem als Objekt psychologischer Kategorisierungsprozeduren, die unweigerlich mit Vorurteilen auf Seiten der Therapeut:innen verwoben seien:

\footnotetext{
1 Bei den Namen handelt es sich um Pseudonyme, um die Anonymität der Interviewpartner zu wahren.

2 Die Buchstaben in den Klammern zeigen die übergeordnete Kategorisierung des Reintegrationsverlaufs an: (a) eine kontinuierlich stabilisierende Entwicklung, (b) eine begrenzt stabilisierende Entwicklung und (c) eine stagnierende Entwicklung.
} 
Es kam häufig vor, dass ich dieses Gefühl hatte, je nachdem, welche Tests sie zum Beispiel machen mussten. Dies zeigte mir, dass ich nichts anderes als eine Nummer bin. [...] Ich weiß, ich werde dadurch, dass ich bereits Scheiße gebaut habe, in meinem Leben schon völlig anders einschubladisiert. Es kann auch jemand kommen und sagen: „Nein, es ist nicht so.“ Aber grundsätzlich bildet sich ein Mensch seine Vorurteile und da kann man noch so sehr behaupten, man sei ein Profi, das mit den Vorurteilen ist einfach so (I/17). ${ }^{3}$

Solche Stigmatisierungserfahrungen im Kontext therapeutischer Diagnostik, die sich teils auf eher subtile Weise vermitteln, werden durch Erzählungen ergänzt, in denen Situationen beschrieben werden, die in ihrer Grundstruktur an Degradierungszeremonien erinnern. Michael (b) führt hierzu aus:

Ich bin recht kaputt gegangen psychisch, [...] eben wegen so Heime, nicht was ich gemacht habe, das habe ich können gut verarbeiten, aber wegen dem Heim selber, wie sie mich täglich oder fast täglich nur runtergemacht haben [...] und runtergedrückt haben, und gesagt haben: „Mit dem schaffst du nie etwas, du wirst ein Junkie“ (II/4).

In dieser retrospektiven Betrachtung wird ein direkter Zusammenhang zwischen der früher erlebten Behandlung und den aktuellen psychischen Belastungen hergestellt. Während für das Verhalten, das zur Verurteilung geführt habe, eine produktive Verarbeitung konstatiert wird, wird den verbalen Abwertungen und Negativzuschreibungen durch die Fachkräfte eine hohe Relevanz für die aktuelle psychische Verfassung zugerechnet.

Eine weitere Steigerung der Intensität von Stigmatisierungserfahrungen ist in einer Erzählung von Stefan (c) zu erkennen. Er erzählt im Rückblick davon, dass er wegen eines Regelverstoßes für sechs Wochen aus der Maßnahme genommen und ins Gefängnis gesperrt worden sei. Er habe immer noch einen ziemlichen „Hass“ (II/10) auf die Jugendanwaltschaft, die diese Intervention veranlasst habe. Die damit verbundene Negativkategorisierung beschäftige ihn seitdem und verunsichere ihn nachhaltig:

Es ist einfach nochmals die Frage: „Bin ich so schlimm, dass man mich dort reinstecken muss, oder bin ich es nicht?" Es ist einfach immer diese Frage wirklich: „Warum? Wieso?“ Und das ist einfach die, wo mir nie wirklich beantwortet worden ist. Das ist das Schlimme daran. Weil ich verstehe es bis heute nicht und das ist wirklich das. Wenn ich es wenigstens verstanden habe und man es mir richtig erklärt hätte, dann, dann versteht man es. [...] Die Frage ist dann einfach: „Eben wenn, wenn sie mich schon so schlimm hinstellen, wieso sollte ich dann nicht genau so schlimm sein?" Das ist einfach das, wo mir immer durch den Kopf geht. Ich meine, da wirst du behandelt wie ein Schwerverbrecher (II/10-11).

\footnotetext{
3 Die römische Ziffer zeigt an, aus welcher Interviewwelle das Zitat stammt, die indisch-arabische Ziffer verweist auf die Seitenzahl des Transkripts.
} 
Auch hier zeigt sich die Erfahrung einer Fehlkategorisierung, die sich diesmal auf die Schwere eines Regelverstoßes und die daraus abgeleiteten Konsequenzen bezieht. Die Diskrepanz zwischen der offiziellen Definition des sanktionierten Verhaltens und der eigenen Einordnung werden als so eklatant beschrieben, dass als Ausweg eine Verhaltensangleichung an die offizielle Definition als Delinquenter entworfen wird.

Neben diesen Erzählungen über Stigmatisierungserfahrungen innerhalb der Maßnahmeeinrichtungen finden sich noch solche, die sich auf den Kontakt mit Leuten außerhalb der Einrichtungen beziehen. Die Maßnahmezentren sind meist in abgelegenen kleinen Ortschaften angesiedelt, so dass nichtansässige Personen wohl leicht zu identifizieren sind. Stefan (c) beschreibt eine für ihn deutlich spürbare Ablehnung der Einwohner:innen gegenüber der Einrichtung und den darin Untergebrachten:

Das Problem ist dann einfach gewesen, es ist eine kleine Gemeinde und die Gemeinden machen einfach ein riesiges Geschiss: „Diese Kriminellen wollen wir nicht haben." (I/2).

Während diese Einschätzung in der Erzählung auf eine in der Institution zirkulierende Geschichte bezogen wird, also eher auf ein daraus abgeleitetes erwartetes Stigmatisierungspotenzial verweist, schildert Marcel (a) eine konkrete Situation, in der er sich aufgrund seiner Zugehörigkeit zum Adressatenkreis der Einrichtung stigmatisiert fühlte:

[A]ls ich Zigaretten kaufen wollte, [...] habe ich den Lehrausweis gezeigt, dann schaut sie mich völlig schockiert an, ich habe es ihr schon angemerkt gehabt, sie hat mir schon die Zigaretten gegeben und so, aber das ist dann, da fühlt man sich dann auch nicht gerade willkommen. [...] [I]ch habe bei mir das Gefühl, man sieht es mir an, dass ich, wie soll ich sagen, dass ich kriminell gewesen bin oder so (I/11).

Auch in dieser Textpassage wird die Verbindung zwischen der Stigmatisierungserfahrung und der daraus folgenden Verunsicherung hinsichtlich der Zuweisung eines sozialen Status betont. Es zeigt sich aber auch, dass das Attribut „kriminell“ sprachlich in der Vergangenheit verortet wird und somit aus dem aktuellen Selbstentwurf ausgeklammert bleibt.

Insgesamt wird deutlich, dass die jungen Männer den im Kontext der Maßnahme erfahrenen Stigmatisierungen eine hohe Relevanz zumessen. Dies lässt sich dahingehend interpretieren, dass die institutionellen Interventionen scharf in die Selbstbildungsprozesse der jungen Männer eingreifen und dadurch gegebenenfalls frühere Stigmatisierungserfahrungen reaktiviert und zugespitzt werden. Vor diesem Hintergrund erscheint es auch plausibel, dass externalisierende Erklärungen der eigenen Entwicklung, etwa in Form von Schuldzuweisungen an das Umfeld, eine entlastende Funktion für die Betroffenen entfalten können. 


\subsubsection{Maßnahmen zur Behandlung von psychischen Störungen sowie Suchtbehandlung}

Für strafrechtlich verurteilte Personen gibt es in der Schweiz die Möglichkeit, dass eine Haftstrafe zugunsten einer therapeutischen Maßnahme zurückgestellt wird, wenn eine psychische Störung oder eine Suchterkrankung diagnostiziert und als behandelbar eingestuft wurde. In Bezug auf Maßnahmen zur Suchtbehandlung finden sich in den meisten Fällen keine oder nur begrenzte Schilderungen von Stigmatisierungserfahrungen. Wenn, dann setzen Stigmatisierungserfahrungen hier zumeist auf einer allgemeineren Ebene an, wie Christian (c) veranschaulicht:

Ich versuche Ihnen das aus meiner Sicht zu erklären, wie es einem zumute ist, wenn einer all das erlebt hat und irgendwo sich, irgendwo diskriminiert fühlt. Wenn man, wenn man halt süchtig ist, heißt dies grad: „Immer süchtig, immer kriminell.“ Oder? Man muss nicht unbedingt kriminell sein, wenn man süchtig ist und man kann auch friedlich sein, man kann auch straffrei leben, wenn man süchtig ist und das geht auch (I/5).

In dieser Aussage wird die unhinterfragte und weitverbreitete Gleichsetzung von Sucht und Kriminalität kritisiert. Solch pauschale Zuschreibungen werden als bedeutsamer Teil des eigenen Erfahrungszusammenhangs präsentiert und für die eigene Person als Fehlkategorisierung zurückgewiesen. Bemerkenswert ist aber, dass diese Zurückweisung nicht auf die Kategorisierung als „süchtig“ bezogen wird. Dies kann als Hinweis darauf gelesen werden, dass dieser Zuschreibungskategorie auch ein gewisser Wert zuerkannt wird, etwa weil sie den Zugang zum geschützten Raum einer betreuten Wohneinrichtung und zu weiteren wichtigen Unterstützungsleistungen ermöglicht.

Maßnahmen aufgrund von psychischen Störungen sind dahingehend ein Sonderfall, dass sie nach fünf Jahren noch einmal um bis zu weitere fünf Jahre verlängert werden können. Bei einer schlechten Prognose hinsichtlich weiterer Straffälligkeit besteht auch die Möglichkeit einer darüberhinausgehenden Verwahrung. Karls (c) Erzählungen sind in weiten Teilen durch seine negativen Erfahrungen mit diesem System geprägt und sehr emotional vorgetragen, da er selbst mehrere Jahre über die eigentliche Unterbringungsdauer hinaus in der Maßnahme untergebracht worden sei. Er erzählt darüber, dass er sich wie ein „Schulbub“ (II/6) gefühlt habe, weil er zu einer bestimmten Zeit am Abend habe in der Einrichtung sein müssen und einen Antrag habe stellen müssen, um ein Mobiltelefon zu bekommen. Für die Freigänge habe er einen Ausweis bekommen, in dem sein Sonderstatus und die erlaubten Ausgangszeiten aufgelistet waren, und habe diese bei polizeilichen Personenkontrollen wiederholt vorzeigen müssen. Dabei habe er sich immer als „,Mensch zweiter Klasse“ (II/6) gefühlt, dem ständig misstraut worden sei: „Wir sind ja immer nur Lügner und Betrüger“ (II/6). Zudem sei er eigentlich als „moderat bis gering gefährlich“ (II/2) eingestuft worden und habe dann aus ihm undurchsichtigen Gründen plötzlich den „Gemeingefährlichkeitsstempel“ (II/3) bekommen. In der Folge habe man ihn, als er sich einmal verspätet habe, sofort ausgeschrieben und das ,Spezialkommando“ (II/3) nach ihm geschickt. Er fühlt sich insgesamt falsch kategorisiert, weil er im Gegensatz zu anderen eigentlich keine Gefahr für die Allgemeinheit darstelle: 
Vergewaltiger und so Pädos und so, die musst du wirklich drin behalten und nicht herumlaufen lassen. Also, einfach im geschlossenen Rahmen, aber nicht im offenen Rahmen, oder. Und die, wo es wirklich kapiert haben, die behalten sie drin (II/6).

Am Ende habe sich für ihn herausgestellt, dass die Verlängerung der Maßnahme „nur eine verdammte scheiß Schikane“ (II/15) gewesen sei, denn auf einmal sei die Maßnahme ohne erkennbaren Grund von einem auf den anderen Tag beendet worden:

Ich fühle mich völlig verarscht, ehrlich gesagt. [...] Zwei Jahre oder vier Jahre, wo man mir jetzt geklaut hat vom Leben, oder? Wer gibt mir das wieder?

(II/15).

Dieses Beispiel zeigt besonders deutlich, wie sich die als unpassend empfundene Zuschreibung des Status, eine Gefahr für die Öffentlichkeit zu sein, mit einer emotionalen Unruhe verbinden kann. Diese affektive Betroffenheit äußert sich auch durch moralische Abgrenzungen gegenüber anderen stigmatisierten Gruppen (vgl. Zahradnik 2020). Die im Strafgesetz systematisch implementierte Unsicherheit bezüglich einer jederzeit möglichen verschärften Kategorisierung und daraus resultierenden längeren Verwahrung schränkt das Subjekt hinsichtlich der Planbarkeit seiner Zukunft massiv ein.

\subsection{Stigmatisierungserfahrungen nach der Entlassung aus dem Vollzug}

\subsubsection{Aufsicht durch die Bewährungsdienste}

Bei einer frühzeitigen Entlassung aus der Haft oder nach einer Maßnahme aufgrund einer psychischen Störung oder zur Suchtbehandlung wird im Regelfall eine Bewährungszeit angeordnet, in der regelmäßige Kontrollen durch die Justizbehörden stattfinden. In einem Großteil der Fälle werden in diesem Zusammenhang keine Stigmatisierungserfahrungen geschildert, vielmehr wird öfter bemängelt, dass die erhoffte praktische Unterstützung der Dienste ausgeblieben sei. In einigen Fällen aber wird auch eine ausgeprägte Zufriedenheit mit der Begleitung geäußert (Rieker 2017).

Im Fall von Thomas (b) spielen die negativen Erfahrungen mit seiner Bewährungshelferin eine zentrale Rolle in seinen Erzählungen und beschäftigen ihn über mehrere Jahre hinweg (vgl. Zahradnik et al. 2019). Im ersten Interview erzählt er, dass er aus einer Maßnahme zur Suchtbehandlung vorzeitig entlassen und dann unter die Aufsicht der Bewährungsdienste gestellt worden sei. Von seiner Bewährungshelferin habe er statt der anfangs versprochenen Hilfe dann aber nur Kontrolle bekommen, vor allem nachdem er einmal konsumiert habe und positiv getestet worden sei. Im nächsten Schritt habe die Bewährungshelferin dann veranlasst, dass auf seine Akte, die sie auch dem Verkehrsamt weitergeleitet habe, der „Stempel: ,Untherapierbar“" (I/12) gekommen sei, was er als klare Fehlkategorisierung empfindet. 
Auch im zweiten Interview nimmt diese Geschichte wieder breiten Raum ein und wird mit Entrüstung weiter ausgeführt. Die Folgen in Form von wiederholten Haarproben, die viel Geld kosten, seien auch aktuell noch deutlich spürbar:

Und wie gesagt, es tut mir jetzt noch äh zwei Jahre nachdem ich aus der Bewährung entlassen bin, tut es mir immer noch schaden. Und äh sie hat ja dazumal nochmals etwas gemacht. Sie hat auf meine Akte drauf geschrieben: „Nicht therapierbar" [...] Ich weiß nicht, ob ich ihre persönlichen Gefühle verletzt habe oder was ich genau dieser Frau gemacht habe, aber ähm sie hat mir ähm recht viel geschadet (II/2).

In dieser Textpassage zeigt sich, dass eine aktenmäßig fixierte Kategorisierung als nachhaltig schädigend empfunden wird. Ein konflikthaftes Moment lässt sich auch darin erkennen, dass Thomas (b) sich selbst auf einem guten Weg hinsichtlich seiner Drogenproblematik sieht und dies mit erkennbarem Stolz erzählt:

Wenn man so sagen will, ich habe eine 25-jährige Suchtgeschichte, ich habe in den letzten zwei Jahren äh genau zwei Abstürze gehabt, Absturzrückfälle, Einzelkonsum. Habe alle meine, mein Know-how angewendet, um etwas dagegen zu machen. Also meiner Ansicht nach bin ich sehr, ja sehr weit äh sehr weit gewachsen (II/2).

Der an sich selbst festgestellte positive Wandel sei von der Bewährungshelferin allerdings nicht erkannt worden. Die Diskrepanz zwischen Selbst- und Fremdkategorisierung besteht vor diesem Hintergrund weiter.

Auch im dritten Interview wird die Verbitterung wegen der Intervention der Bewährungshelferin deutlich. Da weiterhin die Kosten für die angeordneten Haarproben in Raten abbezahlt werden müssen, werde er immer wieder an diese Geschichte erinnert. Er meint, dass ihr Vorgehen, ,so ne Vorverurteilung dahin[zu]schicken“ (III/2), illegal gewesen sei und fühlt sich von ihr zu Unrecht „denunziert“ (III/5).

Im vierten Interview ist das Thema immer noch virulent, da trotz guter Testergebnisse keine Lockerungen erreicht werden könnten. Er müsse bei den andauernden Testprozeduren auch immer wieder an die Bewährungshelferin denken, mittlerweile fühle sich das für ihn wie ein „halbes Trauma“ (IV/3) an. Thomas (b) lehnt das bestehende System aber nicht grundsätzlich ab, sondern kann ihm auch positive Aspekte abgewinnen. Er denkt aber, dass er einer Gruppe zugerechnet wird, die strukturell benachteiligt wird:

Der Schweizer Staat ist nicht nur scheiße und das System ist auch nicht nur scheiße, sondern das System ist so wie es ist und hat sicher seine guten Punkte. Vielleicht hab ich durch meine Situation das Pech gehabt, in einer Minderheit zu sein, so Drogenabhängige, dass ich so ein bisschen mit diesen Punkten in Kontakt gekommen bin, wo man nicht die positiven Seiten des Systems dann sieht (IV/4).

Hier zeigt sich eine Verarbeitungsweise von Stigmatisierungserfahrungen, die auf das Suchen nach einem versöhnlichen Abschluss hindeutet. Es entsteht dabei aber auch der Eindruck, dass mit dem Verblassen der eigenen Betroffenheit auch 
die Kritik an den strukturellen Diskriminierungsrisiken von sozialen Gruppen wie „Drogenabhängige[n]“ an Relevanz verliert.

\subsubsection{Integration in Arbeit}

Der Integration in den ersten Arbeitsmarkt wird im weiteren Fortgang des Wiedereingliederungsprozesses eine hohe Bedeutung zugemessen, aber zumeist werden dabei schwer zu überwindende Barrieren gesehen. Dies gilt vor allem für Einträge im Strafregister, aber auch für fehlende Berufserfahrung sowie ein fortgeschrittenes Alter.

Einträge im Strafregister als Hürde Weit verbreitet ist die Einschätzung, dass Absagen oder ausbleibende Rückmeldungen auf eingereichte Bewerbungen aufgrund der strafrechtlichen Verurteilung erfolgt seien. So sehen manche der jungen Männer, die ihre Ausbildung im Rahmen einer Maßnahme absolviert haben, in dem Umstand, dass im Berufsabschlusszeugnis der Name der Maßnahmeeinrichtung aufgeführt ist, einen entscheidenden Makel. Michael (b) ist auch mehrere Jahre nach der Unterbringung der Ansicht, dass viele Ablehnungen seiner Bewerbungen darauf beruhen würden:

Viele lesen nur: „, [Maßnahme], ah er ist im Heim gewesen. “ Und das ist schon nicht gut. So habe ich viel weniger Chancen (IV/21).

Die Nachfrage des Interviewers, ob es konkrete Diskriminierungssituationen gegeben habe, verneint er aber.

Die am weitesten verbreitete Hürde bei der Arbeitssuche wird von den Befragten in den Einträgen im Strafregister gesehen. Es handelt sich aber hierbei zumeist nicht um eine Erfahrung, die von den Befragten an konkret erlebten Situationen festgemacht, sondern von ihnen vielmehr gedanklich vorweggenommen wird. So entgegnet Klaus (a), einer der älteren Interviewpartner, auf die Frage des Interviewers, ob er sich nicht vorstellen könnte, sich wieder bei seinem früheren Arbeitgeber zu bewerben, kurz und knapp: „Die wollen einen Strafregisterauszug“ (I/3). Sebastian (b) schildert, wie unwohl er sich immer wieder wegen seiner Einträge beim Bewerben gefühlt habe:

Was auch ein Hindernis gewesen ist, ist halt mein Strafregisterauszug. Das ist eine Realität, oder? Und das ist auch äh, ich habe auch gezittert, wo ich mich beworben habe. [...] Heutzutage ist es einfach so, dass man äh gut und gern einen Strafregisterauszug immer wieder mal bringen muss, oder? Und ich habe äh immer noch ein scheues Zittern, wenn ich an das denke (II/13).

Als belastend empfindet er, dass er grundsätzlich von Stellen im öffentlichen Bereich ausgeschlossen sei, denn für ihn als aktenkundigen „Ex-Junkie“ (II/14) sei es quasi unmöglich, dort eine Stelle zu finden. Er bemängelt darüber hinaus die Hartnäckigkeit solcher Stereotypisierungen, durch die bestimmten Personen positive Entwicklungen von Grund auf abgesprochen würden: 
Heutzutage ist es wirklich schwierig, wenn die Leute hören: „Ah, hast du Drogen genommen.“ Dann bist du einfach schon mal unten durch: ,Ah, bist du kriminell gewesen.“ Oder der Stempel: „Einmal kriminell, immer kriminell.“ Was man einfach vergisst ist, dass sich die Menschen auch ändern können, wenn sie wollen (II/15).

Im dritten Interview schildert er anhand einer Bewerbungssituation, wie belastend der Strafregisterauszug für ihn immer noch sei:

Ich habe mich bei einer Bank beworben. Ich bin prompt ans Vorstellungsgespräch gekommen. Ich bin so am Flattern gewesen. Ich habe mich so verstellt. Ich bin so unauthentisch gewesen. [...] Ich bin einfach so unsicher gewesen, dass ich den Strafregisterauszug bringen muss. Ich habe gewusst, ich muss ihn bringen. Darum, die Gesellschaft vergisst nicht so schnell (III/18).

Diese Langwierigkeit einer Statusfestschreibung im Strafregister beschäftigt auch Rolf (c), der seine langjährige Inhaftierung als so hohe Hürde ansieht, dass er für sich keine seiner Ausbildung angemessenen Erwerbsarbeitsoptionen mehr sieht:

Das ganze Berufliche ist zusammengebrochen, ich hatte eine Verurteilung wegen eines Kapitaldelikts, ich muss nie mehr in meinem Leben eine Karriere anstreben, weil das steht jetzt noch über zehn Jahre in meinem Strafregister drinnen und wenn ich mich für eine Stelle bewerbe und ein Strafregisterauszug verlangt wird, dann werde ich nie mehr eine Stelle bekommen, die meiner Ausbildung entspricht. Und mit dem habe ich mich schon abgefunden (I/4).

Die Stigmatisierung aufgrund seiner strafrechtlich sanktionierten Vergehen spüre er aber nicht nur in Bezug auf die Arbeitssuche, sondern bekomme sie auch direkt von dem Berufsverband zu spüren, dem er schon Jahrzehnte angehöre. Dort sei darüber abgestimmt worden, ob man ihn als Mitglied behalten wolle und schließlich sei man zu dem Ergebnis gekommen, dass er einen passiven Status erhalte, bis die Eintragung aus seinem Strafregister gelöscht sei.

Fortgeschrittenes Alter als zusätzliche Belastung Bei manchen der Interviewten zeigt sich ein kumulierender Effekt verschiedener Stigmatisierungsbefürchtungen. So ist z. B. Herbert (a) davon überzeugt, dass neben den durch seine Vergangenheit im Drogenmilieu sowie der damit verbundenen Verurteilungen entstandenen Lücken in seinem Lebenslauf nun auch sein fortgeschrittenes Alter eine entscheidende Rolle spielen würde:

Weil ich bekomme sonst nichts. [...] Es ist so schwierig. Es ist fast ein Ding der, in meinem Alter, mit meiner Vergangenheit. [...] Und jetzt wieder Bewerbungen machen mit irgend Zeugnissen, wo du zehn, fünfzehn Jahre Lücken hast oder so (II/3).

Für Ludwig (b) spielt der Führerschein, den er gerade zu machen versuche, eine wichtige Rolle in Hinblick auf die Arbeitsintegration. In dem Fall, dass er ihn nicht bekomme, sieht er aufgrund seines Alters nur geringe Chancen für eine Anstellung im ersten Arbeitsmarkt: 
Nehmen wir mal an, ich bekomme keinen Fahrausweis. Dann wird es in meinem Alter und mit meiner Vergangenheit sehr schwierig regulär einen Job zu finden. In dem Fall könnte ich auch mit Freiwilligenarbeit beginnen und mich so bewähren, dass gesehen wird, dass ich wirklich will und auch die Energie dazu habe beziehungsweise trotz allem noch etwas aus meinem Leben machen möchte (I/5).

Ludwig (b) zeigt sich hier entschlossen, der gesellschaftlich geforderten Arbeitsorientierung auch durch unbezahlte Arbeit nachzukommen und dadurch nach außen ein positives Zeichen setzen zu können. Im zweiten Interview erzählt er, dass er sich gemeinsam mit seiner Bewährungshelferin überlegt habe, eine Stiftung um finanzielle Unterstützung für den Führerschein zu bitten. Um dem Makel, den er aufgrund seines früheren Drogenkonsums als gegeben ansieht, zu begegnen, begrüßt er ihre Bereitschaft, ihm ein Leumundszeugnis auszustellen:

Sie würde auch gerne noch einen Brief dazulegen, in dem sie dann die Sicht von der Justiz darlege, und dass das wirklich eine sinnvolle Sache sei und nicht, dass die dann das Gefühl haben, sie müssen irgendwie einem Ex-Junkie ein paar Tausend Franken überweisen (II/11).

Zum Zeitpunkt des dritten Interviews konnte weder der Führerschein abgeschlossen noch eine Stelle gefunden werden und das Alter wird weiterhin als entscheidende Integrationshürde angesehen: „Ich denke, es wird eh schwierig in meinem Alter, ich mein, ich bin jetzt 53, dass mich noch irgendjemand einstellt“ (III/1).

Solche kumulierten Stigmatisierungserfahrungen werden vor allem von älteren Männern im Sample geschildert, die aufgrund von langen Phasen intensiven Drogenkonsums eine gewisse Ferne zum ersten Arbeitsmarkt aufweisen. Stellenweise können aber dennoch Fortschritte hinsichtlich der Arbeitsintegration erreicht werden, etwa wenn sich Beschäftigungsmöglichkeiten finden, die speziell auf ältere Personen mit einem solchen Hintergrund ausgerichtet sind oder aber diesen Merkmalen von Arbeitgeber:innen keine besondere Bedeutung zugemessen wird.

Umgangsweisen mit einer strafrechtlichen Verurteilung Eine weitverbreitete Strategie im Umgang mit Zeiträumen der strafrechtlich angeordneten Unterbringung ist das Kaschieren derselben im Lebenslauf. Dies werde teilweise auch von Seiten beratender Stellen wie z. B. den Sozialdiensten in Wohneinrichtungen empfohlen, um die Chancen am Arbeitsmarkt zu erhöhen. So berichtet Roland (c): „Ob ich voll offene Karten gemacht habe? Nein, kann ich ihnen gerade äh so zugeben, nein natürlich nicht“ (II/5). Ähnliches erzählt auch Fynn (a), der die Zeit in der Maßnahme für Jugendliche nicht offenlegen möchte:

Ich habe ein wenig gelogen in der Bewerbung einfach. Weil das mit der [Maßnahme], das kommt mir sehr schräg rüber, wenn ich das auch reinschreibe. Ich habe einfach geschrieben, dass ich ein Jahr auf dem Sprachaufenthalt in [Land] gewesen bin, statt einfach [Maßnahmezentrum] (II/3).

Ulrich (a) hingegen berichtet, dass die Regionale Arbeitsvermittlung (RAV) ihm eine solche Vorgehensweise untersagt habe: 
Ich habe ja versucht meine Bewerbungen ein wenig, wie soll ich jetzt sagen, ein wenig zu fälschen, oder? Da bin ich beim RAV gewesen, oder? Und die haben das gemerkt, haben mich bestraft dafür und ich musste dann reinschreiben, dass ich in Gefangenschaft war und das ist natürlich fatal [...]. Ja und jetzt habe ich das einfach in meinen Bewerbungen drin, oder, und das ist halt schon kein guter Punkt (II/7).

Ihm sei es dann aber trotz Offenlegung der Vergangenheit gelungen, eine Stelle zu finden, diese Offenlegung habe sich aber danach als verunsichernd und beschränkend ausgewirkt. Er habe seinen Chef von Anfang an über seine früheren Straftaten in Kenntnis gesetzt, nun aber das Gefühl, dass er deswegen schlechter bezahlt und behandelt werde als die Kollegen:

Habe dort, ja eigentlich seit Anfang, Probleme mit meinem Arbeitgeber und zwar was das Zwischenmenschliche angeht. Er behandelt mich einfach nicht so wie die andern (II/2).

Der Umgang mit der strafrechtlichen Verurteilung nach einer gelungenen Arbeitsintegration stellt sich zum Teil auch nach längerer Zeit noch als herausfordernd dar. Thomas (b), der nach einer justiziell verordneten Drogentherapie noch mit Methadon substituiert, absolviert eine Ausbildung im Pflegebereich und erzählt davon, wie er sich vor negativen Zuschreibungen zu schützen versucht. Während es ihm bisher ein Anliegen gewesen sei, offen mit seiner Geschichte umzugehen, habe er seine Strategie nun geändert:

Mein neues Arbeitsumfeld weiß nichts davon. [...] Als ich die Stelle suchte, habe ich's nicht mehr gesagt, weil ich mir auch gesagt hab: „Okey, hier bin ich, ich nehm keine Drogen, [...] die tun mir nichts mehr, also ich bin nicht anders, weil ich das mal genommen hab, als einer der's nicht genommen hat in meinem Empfinden und deshalb ist es auch nicht die erste Info, die ich euch geben werde." Nur, damit ich dann schön jedes Mal, wenn ich ne Erkältung habe, einen Rückfall hatte oder sonst irgend ne schräge Geschichte. Oder wenn mal 'n Portemonnaie fehlt auf der Abteilung: „Ähhh, der Ex-Junkie“, also einfach so bisschen vorzubeugen, dass ich da so in ein Klischee reinstürze, habe ich mich dazu entschlossen, einen anderen Umgang damit zu finden. Ja, das ist mir auch gut gelungen (III/6).

Im vierten Interview geht Thomas (b) noch einmal auf seinen Umgang mit seiner Vergangenheit im Arbeitskontext ein und wie sich dieser etwas entspannt habe. Er habe den Eindruck, dass er die „Suchtvergangenheit [...] nicht mehr repräsentiere“ (IV/6) und nun mit dem zeitlichen Abstand auch eine ,gewisse Sicherheit“ (IV/6) entwickelt habe:

Mittlerweile erzähle ich davon, ich gebe aber niemals Quellen an, da mache ich ein Geheimnis darum herum. Deshalb muss ich sagen, fühle ich mich nicht mehr so stigmatisiert, weil ich wirklich das Glück habe, in einem normalen Alltag funktionieren zu können. [...] Und darum, ja, fühle ich mich nicht mehr so wie früher, also Randgruppe, ausgeschlossen (IV/6-7). 
Insgesamt zeigt sich in den Erzählungen, dass Stigmatisierungserfahrungen im Kontext von Arbeit häufig in Form von Vermutungen oder Erwartungen geäußert und seltener auf konkret erlebte Situationen bezogen werden. Dies kann als Hinweis darauf interpretiert werden, dass der Zugang zum Arbeitsmarkt für strafrechtlich verurteilte Personen eher durch Mechanismen einer verdeckten strukturellen Diskriminierung erschwert wird, die sich nicht einfach erkennen lassen und denen man nur schwer ausweichen kann. Am verbreitetsten ist deshalb die Strategie, genauere Informationen über die Vergangenheit entweder zu verschweigen oder zumindest in veränderter Form zu kommunizieren.

\subsubsection{Stigmatisierungserfahrungen in anderen Kontexten}

Stigmatisierungserfahrungen werden auch in Bezug auf weitere reintegrationsrelevante Kontexte thematisiert. Während Negativzuschreibungen bei der Wohnungssuche zumeist auch eher in Form von Vermutungen geäußert werden, treten sie im Kontakt mit Behörden oder in Bezug auf Beziehungen meist deutlicher zutage.

Wohnungssuche Eine strafrechtliche Verurteilung wird von manchen Interviewpartnern als entscheidende Hürde bei der Wohnungssuche angesehen. Klaus (a) etwa ist der Meinung, dass es einen wichtigen Unterschied mache, welche Verwaltung hinter der Vergabe von Wohnungen stehe. Bei ihm habe schließlich die Unterstuitzung der Bewährungshelferin den Ausschlag für eine Zusage gegeben:

Es ist ein Familienbetrieb, das ist der Vorteil. Bei großen Verwaltungen haben wir keine Chancen mehr, wenn man aus dem Gefängnis kommt, oder? Gut, sie hat nachher mit denen da telefoniert und auf das hin habe ich die Wohnung bekommen (II/5).

Die Wohnungssuche kann noch durch weitere Faktoren wie etwa Schulden und Einträge im Betreibungsregister als erschwert erlebt werden. Holger (b) etwa erzählt, dass er schon eine lange Suche hinter sich habe, die ihn zunehmend belaste, zumal er einen kleinen Sohn habe, um den er sich alleine kümmern müsse: „Stress. Ich meine, such einmal eine Wohnung mit dreissig-, vierzigtausend Franken Schulden als ExHäftling“ (I/15). Ludwig (b) berichtet ebenfalls von umfassenden Suchbemühungen, die bisher erfolglos geblieben seien:

Ich habe, ich weiß auch nicht, etwa sechzig Absagen zu Hause bereits. Gut, eben, aber das sind alles noch, das sind die, bei denen ich noch den Eintrag im Betreibungsregisterauszug drin habe (II/11).

Wichtig bei der Wohnungssuche seien zudem Referenzen, die man bei einer Bewerbung zur Absicherung angeben könne. Hierbei könne es entscheidend sein, welcher Status hierdurch vermittelt werde. Ludwig (b), der einen geförderten Arbeitsplatz in der Werkstatt einer betreuten Wohneinrichtung hat, erzählt, dass er lange Zeit das Sozialamt als Sicherheit angegeben habe, aber ,[v]iele sagen einfach: ,Wir wollen keine Sozialfälle“" (III/6). Nachdem er sich einmal mit seinem Chef darüber unterhalten habe, habe ihm dieser geraten, das Sozialamt nicht zu erwähnen und stattdessen ihn als Referenz anzugeben. Daraufhin habe die Verwaltung 
bei seinem Chef angerufen, woraufhin er die Wohnung bekommen habe. Hier zeigt sich, welch hohe Relevanz dem Wissen über die Strukturen des Wohnungsmarktes sowie den richtigen Beziehungen zugemessen wird, wenn man aus einer sozial marginalisierten Position heraus agieren muss.

Behördenkontakte Nach einer Haftstrafe oder der Unterbringung in einer Maßnahme kommt es häufig dazu, dass Sozialleistungen beantragt werden müssen, um den Lebensunterhalt zu sichern. Zum einen können Leistungen der Sozialhilfe beantragt werden, zum anderen gibt es bei gesundheitlichen Einschränkungen die Möglichkeit, von der Invalidenversicherung unterstützt zu werden. Während im Zusammenhang mit dem Sozialamt keine negativen Erlebnisse aufgrund der strafrechtlichen oder drogenbezogenen Vergangenheit geschildert werden, werden bezüglich der Invalidenversicherung häufiger auch stigmatisierende Situationen beschrieben.

Herbert (a) erzählt von seinen Erfahrungen mit der Invalidenversicherung (IV), dass dort nach mehreren Jahren des Leistungsbezugs ein unerwarteter Drogentest angeordnet worden sei, weil er gegenüber der Amtsärztin zu offen mit seinen Konsumgewohnheiten umgegangen sei. Da der Test positiv ausfiel, seien ihm weitere Urinproben zur Überprüfung seines Konsums auferlegt worden:

Dann kam die IV und dort haben sie mich dann wieder fertig gemacht, [...] sogar der Psychiater hat gesagt: „Sie sind zu ehrlich, sie dürfen bei der Ärztin nicht so ehrlich sein.“ (III/7).

Thomas (b) erzählt, dass er trotz der aus seiner Sicht gelungenen sozialen Wiedereingliederung im Behördenkontext wiederkehrend mit Negativzuschreibungen konfrontiert werde und sich ,schikaniert“ (IV/14) fühle:

Immer, wenn ich wieder mit Behörden in Kontakt komme, dann merke ich wieder, dass das da war. Dann geht es so zack zack und dann bin ich wieder reduziert auf ja: „Früher mal Drogen genommen, selbstverschuldet.“ Alles das. Sonst im täglichen Leben nicht so (IV/7).

Insgesamt fällt bei den Behördenkontakten auf, dass Stigmatisierungserfahrungen hier eng mit drogenbezogenen Kategorisierungen verbunden sind. Folgt man den Erzählungen, dann werden gesundheitliche Einschränkungen, die auf einen intensiven Drogenkonsum zurückgeführt werden, in diesem Kontext in individualisierender Weise als Selbstschädigung zugeschrieben und strukturelle Ursachen tendenziell ausgeblendet (vgl. Zahradnik 2020).

Soziale Kontakte und Beziehungen Auch im Kontext von sozialen Kontakten und Beziehungen werden vereinzelt Stigmatisierungserfahrungen aufgrund der strafrechtlichen und drogenbezogenen Vergangenheit berichtet. Patrick (a) etwa erzählt von einer Zurückweisung durch eine Frau, die er kennengelernt habe:

[D]ann sagt sie mir so: „Du hättest mir auch sagen können, dass du kriminell gewesen bist." Die hat durch irgendwelche Leute erfahren, dass ich im Gefängnis gewesen bin und dann hat sie gesagt, dass sie mit mir nichts zu tun haben will, so bäm, abgebrochen (I/10). 
Toni (c) schildert eine Situation, in der ein langjähriger Freund den Kontakt mit ihm beendet habe, weil er mit seiner Geschichte nicht mehr zurechtgekommen sei:

[U]nd ich habe ja schon einen Kumpel gehabt, wo mir nach 15 Jahren die Freundschaft gekündigt hat, weil er gefunden hat: „Ja Toni, ich komme mit deiner Vergangenheit nicht zurecht, mit deinem Rucksack, wo du auf dir trägst.“ (III/14).

Sebastian (b) erzählt davon, dass er bei einer öffentlichen Veranstaltung von einem alten Bekannten wegen seiner Vergangenheit vorgeführt worden sei:

Und die Gesellschaft vergisst nicht. [...] Ein alter Kollege von mir, von früher habe ich getroffen. Und der hat mich voll, hat mich voll äh bloßgestellt vor, vor fremden Leuten hat er gesagt: „Ja weißt du, [...] das letzte Mal als ich dich gesehen habe, bist du mit so einem [...] Holzlöffel rumgelaufen im [Park] und hast so die Leute angebettelt, ob sie irgendwas in deine Holzkelle reinwerfen.“ (III/19-20).

Während die Stigmatisierungserfahrungen in den bisher beschriebenen Textpassagen eher punktuelle Erlebnisse umreißen, zeigt sich bei Mike (a) eine nachhaltigere Belastung durch die Kontaktvermeidung seines früheren sozialen Umfeldes. Trotz seiner intensiven und erfolgreichen Reintegrationsbemühungen im Arbeitsbereich würden sich für ihn einst wichtige Bezugspersonen dauerhaft von ihm distanzieren:

Viele Freunde von mir von früher [...], haben mir den Rücken zugedreht. Wirklich also, und ich rede da von wirklich guten Freunden von mir von früher. Entweder wollen sie nichts mehr mit mir zu tun haben oder äh haben irgendwelche Ausreden, immer wenn ich mit ihnen will abmachen (III/10).

Die erfahrenen Zurückweisungen hätten dann auch zur Folge, dass er sich zunehmend isoliert fühle und sich zurückziehe:

Es ist schon belastend. Ich fühle mich zum Teil äh, muss ich Ihnen ganz ehrlich sagen, zum Teil fühle ich mich einsam zu Hause, mir ist langweilig, ja. [...] Aber trotzdem würde ich gerne wieder mal mit einem von früher wieder gehen, wieder eins gehen trinken. Aber dann heißt es: „Ja schau, jetzt gehst du mit dem Drögeler, mit dem, wo im Gefängnis gewesen ist.“ [...] Ich bin voll abgestempelt, egal jetzt äh, ob das jetzt von einem System her ist, also von der Gesellschaft, oder von den Kollegen (III/11).

Das Gefühl der sozialen Isolation ist auch im vierten Interview noch sehr präsent und es ergibt sich der Eindruck, dass sich eine gewisse Resignation eingestellt hat:

Ah ja, ich habe viele, ist auch jetzt noch so, dass viele meiner alten Freunde Abstand nehmen von mir, ja. Also das ist immer noch so. Leider Gottes (IV/19).

Es wird schließlich eine Müdigkeit konstatiert, weiterhin auf die früheren Freunde zuzugehen und sich für die Vergangenheit rechtfertigen zu müssen:

Ja, ich habe einfach keine Lust, mit Leuten die ganzen Geschichten da durchzukauen und warum da wieso und wieso ist es passiert. Ich meine, ich habe 
mit den meisten ja darüber gesprochen. [...] Es ist schwerer von der schlechten

Seite wieder auf die gute zu kommen als umgekehrt (IV/19).

Mit dem letzten Satz fasst Mike (a) noch einmal die Schwierigkeit zusammen, vergangene Fehltritte in Beziehungen zu glätten und verlorenes Vertrauen wiederaufzubauen. Hier wird insgesamt deutlich, wie belastend das Gefühl einer langandauernden Zurückweisung erfahren wird und wie es den Reintegrationsprozess herausfordern kann.

\section{Fazit}

Stigmatisierungen aufgrund einer strafrechtlichen Verurteilung haben für viele unserer Gesprächspartner eine hohe Bedeutung im Reintegrationsprozess und werden von ihnen als einschneidende Erfahrungen beschrieben (vgl. Matt 2014). Wichtig erscheint insbesondere die Unterscheidung zwischen erwarteten und erlebten Stigmatisierungen (vgl. Gurr und Jungbauer-Gans 2017, S. 43). Während vor allem in Bezug auf institutionelle Kontexte sowie im privaten Bereich konkrete negative Zuschreibungssituationen geschildert werden, ist dies im Kontext von Arbeitsund Wohnungssuche eher nicht der Fall. Dort scheinen Selektionsprozesse und die ihnen zugrundeliegenden Bewertungskriterien weniger transparent zu sein, so dass eine Absage interpretationsbedürftig bleibt. Hierbei bilden gesellschaftliche Wissensbestände über strukturelle Diskriminierungszusammenhänge geeignete und wahrscheinlich auch zutreffende Orientierungsfolien für die Einordnung der eigenen Erfahrungen. Vor allem bei Situationen, in denen ein Strafregisterauszug vorgelegt werden muss, ist die Annahme eines solchen Zusammenhangs hoch plausibel. Es ist aber wohl auch davon auszugehen, dass den Betroffenen strukturelle Benachteiligungen nicht immer bewusst werden und sie deshalb nicht darüber berichten. Dies wirft aber methodisch-methodologische Fragen dazu auf, wie solch verdeckt bleibende Stigmatisierungen empirisch aufgeschlüsselt werden könnten.

Die auf konkrete Situationen bezogenen Stigmatisierungserfahrungen in institutionellen Kontexten lassen sich ebenso auf strukturelle Ursachen zurückführen. Hier ist aber davon auszugehen, dass Zuschreibungen kaum verdeckt zu halten sind, da ja die Strukturen der Organisationen darauf ausgelegt sind, dass ihnen Personen aufgrund von bestimmten Kategorisierungen zugewiesen werden und entsprechend bestimmter Programmatiken zu behandeln sind. Dies ist im Regelfall auch den zugewiesenen Personen bekannt. Grundsätzlich ist wohl davon auszugehen, dass zwischen Selbst- und Fremdkategorisierungen eine gewisse Deckung besteht und Konflikte meist nicht offen zutage treten. Subjektive Erfahrungen von Fehlkategorisierungen können sich dann aber vor allem zuspitzen, wenn die Diskrepanz zwischen Selbst- und Fremdkategorisierung über die Zeit bestehen bleibt oder zunimmt.

Solche Dynamiken zeigen sich vor allem dort, wo Stigmatisierungserfahrungen auch ausschnittsweise im Längsschnitt betrachtet werden können. So erweist sich etwa eine aktenfixierte Kategorisierung im Behördenkontext als besonders hartnäckig gegenüber einer sich verändernden Selbstwahrnehmung. Ähnliches ist aber auch in Bezug auf den Versuch einer Wiederannäherung an frühere Freunde zu 
erkennen, deren Blickrichtung festgeschrieben scheint, auch wenn die subjektive Selbsteinschätzung erkennbar in Bewegung geraten ist. Besonders deutlich wird die Stabilität einer äußerlichen kategorialen Zuordnung dort, wo eine längere Phase des intensiven Drogenkonsums einen Teil der Biographie bildet. Die gesellschaftlich verbreitete stereotype Zuschreibung der Kategorie „Junkie“ sowie deren Gleichsetzung mit einer kriminellen Veranlagung erscheint in den Erzählungen als besonders herausforderndes Moment für die Etablierung eines veränderten Selbstbildes (vgl. Conner und Rosen 2008).

Schließlich soll der Blick noch einmal auf den Befund gerichtet werden, dass der Großteil der Stigmatisierungserfahrungen von denjenigen Gesprächspartnern geschildert wurde, die über die vier Befragungswellen hinweg einen tendenziell stabilisierenden Verlauf aufweisen. Hier könnte eine Erklärung darin liegen, dass in dieser Gruppe die Integrationsorientierung besonders ausgeprägt ist und deshalb der soziale Rückzug keine geeignete Option darzustellen scheint. Hiermit könnte auch ein insgesamt proaktiverer Umgang mit der eigenen Vergangenheit und den damit verbundenen Zuschreibungen verbunden sein: Im Sinne von Identitätsarbeit ergibt man sich den Fremdkategorisierungen nicht einfach, sondern versucht den eigenen Selbstentwurf dagegenzuhalten und zu stärken, auch wenn dieses Unterfangen immer wieder herausgefordert und stellenweise durchkreuzt wird.

In der dem Beitrag zugrundeliegenden Studie konnten die Stigmatisierungserfahrungen von strafrechtlich verurteilten Männern in der Schweiz im Längsschnitt über mehrere Jahre und verschiedene soziale Kontexte hinweg untersucht werden. Diese Fokussierung auf die subjektiven Deutungs- und Verarbeitungsweisen im Zeitverlauf kann die bisherige, zumeist im Querschnitt angelegte Stigmatisierungsforschung somit perspektivisch gewinnbringend erweitern. Weiterer Forschungsbedarf in diesem thematischen Bereich besteht noch in der Untersuchung von direkten Interaktionen zwischen diskreditierten und diskreditierenden Personen in den unterschiedlichen Phasen und sozialen Kontexten des Reintegrationsprozesses, um auch den konreten Vollzug von Stigmatisierungen besser verstehen zu können. Im zugrundeliegenden Forschungsprojekt haben wir es zudem als gewinnbringend erachtet, auch Personen, die von den Betroffenen als bedeutsam für den eigenen Reintegrationsprozess beschrieben werden, zu interviewen (vgl. Humm et al. 2022). Die noch austehende Analyse dieser Interviews eröffnet eine zusätzliche Perspektive auf Stigmatisierungserfahrungen.

Danksagung Besonderer Dank gilt meinen Kollegen Peter Rieker und Jakob Humm für Rückmeldungen zu frühen Fassungen des Textes sowie den Gutachter:innen und den Herausgeberinnen für wertvolle Anregungen zur eingereichten Fassung.

Open Access Dieser Artikel wird unter der Creative Commons Namensnennung 4.0 International Lizenz veröffentlicht, welche die Nutzung, Vervielfältigung, Bearbeitung, Verbreitung und Wiedergabe in jeglichem Medium und Format erlaubt, sofern Sie den/die ursprünglichen Autor(en) und die Quelle ordnungsgemäß nennen, einen Link zur Creative Commons Lizenz beifügen und angeben, ob Änderungen vorgenommen wurden.

Die in diesem Artikel enthaltenen Bilder und sonstiges Drittmaterial unterliegen ebenfalls der genannten Creative Commons Lizenz, sofern sich aus der Abbildungslegende nichts anderes ergibt. Sofern das betreffende Material nicht unter der genannten Creative Commons Lizenz steht und die betreffende Handlung 
nicht nach gesetzlichen Vorschriften erlaubt ist, ist für die oben aufgeführten Weiterverwendungen des Materials die Einwilligung des jeweiligen Rechteinhabers einzuholen.

Weitere Details zur Lizenz entnehmen Sie bitte der Lizenzinformation auf http://creativecommons.org/ licenses/by/4.0/deed.de.

\section{Literatur}

Conner, Kyaien O., und Daniel Rosen. 2008. "You're nothing but a junkie": multiple experiences of stigma in an aging methadone maintenance population. Journal of Social Work Practice in the Addictions 8(2):244-264.

Corrigan, Patrick W., und Amy C. Watson. 2002. The paradox of self-stigma and mental illness. Clinical Psychology 9(1):35-53.

Dollinger, Bernd. 2018. Subjects in criminality discourse: On the narrative positioning of young defendants. Punishment \& Society 20(4):477-497.

Dollinger, Bernd. 2021. Problemgeschichten. Implikationen einer narrativen Konzeptualisierung und Analyse sozialer Probleme. Soziale Probleme https://doi.org/10.1007/s41059-020-00069-8.

Fürst, Silke. 2018. „Die Volksseele kocht!“ Eine inhaltsanalytische Untersuchung des Nachrichtenfaktors Öffentlichkeitsresonanz im ,Fall Carlos'. Medien Journal 42(4):55-74.

Goffman, Erving. 1975. Stigma: über Techniken der Bewältigung beschädigter Identität. Frankfurt am Main: Suhrkamp.

Gurr, Thomas, und Monika Jungbauer-Gans. 2017. Eine Untersuchung zu Erfahrungen Betroffener mit dem Stigma Arbeitslosigkeit. Soziale Probleme 28(1):25-50.

Hinterleitner, Markus. 2018. Policy failures, blame games and changes to policy practice. Journal of Public Policy 38(2):221-242.

Hirschauer, Stefan. 2017. Humandifferenzierung. Modi und Grade sozialer Zugehörigkeit. In Un/doing Differences. Praktiken der Humandifferenzierung, Hrsg. Stefan Hirschauer, 29-54. Weilerswist: Velbrück.

Humm, Jakob, Peter Rieker, und Franz Zahradnik. 2022. Von Drinnen nach Draußen - und dann? Reintegration nach einer strafrechtlichen Verurteilung - Ergebnisse einer qualitativen Längsschnittuntersuchung. Weinheim, Basel: Beltz Juventa.

Keene, Danya E., Amy B. Smoyer, und Kim M. Blankenship. 2018. Stigma, housing and identity after prison. The Sociological Review Monographs 66(4):799-815.

LeBel, Thomas P. 2008. Perceptions and responses to stigma. Sociology Compass 2(2):409-432.

LeBel, Thomas P. 2012. "if one Doesn't get you another one Will": formerly incarcerated persons' perceptions of discrimination. The Prison Journal 92(1):63-87.

LeBel, Thomas P., Ros Burnett, Shadd Maruna, und Shawn Bushway. 2008. The 'chicken and egg' of subjective and social factors in desistance from crime. European Journal of Criminology 5(2):131-159.

Link, Bruce G., und Jo C. Phelan. 2001. Conceptualizing stigma. Annual Review of Sociology 27:363-385.

Lucius-Hoene, Gabriele, und Arnulf Deppermann. 2004. Narrative Identität und Positionierung. Gesprächsforschung 5:166-183.

Maruna, Shadd. 2001. Making good. How ex-convicts reform and rebuild their lives. American Psychological Association: Washington, DC.

Matt, Eduard. 2014. Übergangsmanagement und der Ausstieg aus Straffälligkeit - Wiedereingliederung als gemeinschaftliche Aufgabe. Herbolzheim: Centaurus.

Naplava, Thomas. 2008. Jugendliche Intensivtäter als Kriminalitätsproblem und Problemkonstruktion. In Soziologie sozialer Probleme und sozialer Kontrolle - Realitäten, Repräsentationen und Politik, Hrsg. Axel Groenemeyer, Silvia Wieseler, 193-214. Wiesbaden: Springer VS.

Oberwittler, Dietrich. 2012. Kriminalität und Delinquenz als soziales Problem. In Handbuch soziale Probleme, 2. Aufl., Hrsg. Günter Albrecht, Axel Groenemeyer, 772-860. Wiesbaden: Springer VS.

Pager, Devah. 2003. The mark of a criminal record. American Journal of Sociology 108(5):937-975.

Pescosolido, Bernice A., und Jack K. Martin. 2015. The stigma complex. Annual Review of Sociology 41:87-116.

Petersilia, Joan. 2003. When prisoners come home: parole and prisoner reentry. NewYork: Oxford University Press.

Rieker, Peter. 2017. Die soziale Reintegration Straffälliger vor dem Hintergrund ihrer sozialen Beziehungen. Monatsschrift für Kriminologie und Strafrechtsreform 100(6):453-472. 
Rieker, Peter. 2022. Reintegration: Konzept - Varianten - Verläufe. In Von Drinnen nach Draußen - und dann? Reintegration nach einer strafrechtlichen Verurteilung - Ergebnisse einer qualitativen Längsschnittuntersuchung, Hrsg. Jakob Humm, Peter Rieker, und Franz Zahradnik, 34-48. Weinheim, Basel: Beltz Juventa.

Schranz, Mario. 2015. „Carlos“ und die Medien - eine öffentliche Debatte kritisch reflektiert. In Schweizer Jugendstrafrecht: Vorbildlich oder überholt?, Hrsg. Franz Riklin, Bettina Mez, 63-77. Bern: Stämpfli.

Scott, Marvin B., und Stanford M. Lyman. 1968. Accounts. American Sociological Review 33(1):46-62.

Siegel, Karolynn, Howard Lune, und Ilan H. Meyer. 1998. Stigma management among Gay/ bisexual men with HIV/AIDS. Qualitative Sociology 21(1):3-23.

Soeffner, Hans-Georg. 2004. Auslegung des Alltags - Der Alltag der Auslegung, 2. Aufl., Konstanz: UVK.

Stelly, Wolfgang, und Jürgen Thomas. 2004. Wege aus schwerer Jugendkriminalität. Eine qualitative Studie zu Hintergründen und Bedingungen einer erfolgreichen Reintegration von mehrfachauffälligen Jungtätern. Tübingen: Universität Tübingen.

Stenger, Horst. 1985. Stigma und Identität. Über den Umgang straffälliger Jugendlicher mit dem Etikett „kriminell“. Zeitschrift für Soziologie 14(1):28-49.

Strauss, Anselm L. 2007. Grundlagen qualitativer Sozialforschung. Datenanalyse und Theoriebildung in der empirischen soziologischen Forschung. München: Fink.

Sykes, Gresham M., und David Matza. 1957. Techniques of neutralization: a theory of delinquency. American Sociological Review 22(6):664-670.

Visher, Christy A., und Jeremy Travis. 2003. Transitions from prison to community: understanding individual pathways. Annual Review of Sociology 29:89-113.

Western, Bruce, Jeffrey R. Kling, und David F. Weiman. 2001. The labor market consequences of incarceration. Crime and Delinquency 47(3):410-427.

Witzel, Andreas. 2000. Das problemzentrierte Interview [25 Absätze]. Forum Qualitative Sozialforschung 1(1):Art. 22.

Zahradnik, Franz. 2020. Biografische Dynamiken moralischer Positionierungen in Prozessen sozialer Reintegration. Eine Längsschnittanalyse im Kontext von Drogenkonsum, Kriminalität und Arbeit. Journal für Psychologie 28(2):78-100.

Zahradnik, Franz, und Jakob Humm. 2016. Zwischen Aufarbeitung und Befähigung - Integrationskonflikte von Straftätern während und nach einer Vollzugsmaßnahme für junge Erwachsene in der Schweiz. Soziale Probleme 27(2):179-202.

Zahradnik, Franz, und Jakob Humm. 2019. Subjektive Verarbeitungsweisen von Lern- und Erziehungssettings in stationären Maßnahmen für junge Delinquente in der Deutschschweiz. In Bildung und Erziehung im Abseits - Erste Annäherungen, Hrsg. Marcel Schweder, 249-265. Weinheim, Basel: Beltz Juventa.

Zahradnik, Franz, Peter Rieker, und Jakob Humm. 2019. Die Bedeutung persönlicher Beziehungen im Kontext professioneller Hilfe und Kontrolle für die soziale Reintegration verurteilter Straftäter. $B e$ währungshilfe 66(3):252-266. 